\title{
Withdrawn Draft
}

\section{Warning Notice}

The attached draft document has been withdrawn and is provided solely for historical purposes.

It has been superseded by the document identified below.

Withdrawal Date June 24, 2022

Original Release Date February 17, 2022

\section{Superseding Document}

Status Final

Series/Number NIST SP 800-219

Title Automated Secure Configuration Guidance from the macOS Security Compliance Project (mSCP)

Publication Date June 2022

DOI https://doi.org/10.6028/NIST.SP.800-219

CSRC URL https://csrc.nist.gov/publications/detail/sp/800-219/final

\section{Additional Information}


Automated Secure Configuration

Mark Trapnell

Eric Trapnell 
Mark Trapnell

Eric Trapnell

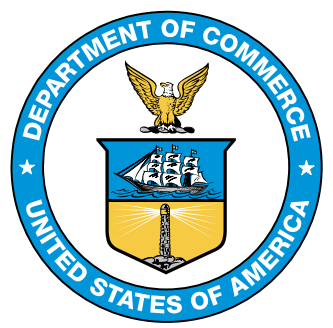

U.S. Department of Commerce Gina M. Raimondo, Secretary

National Institute of Standards and Technology James K. Olthoff, Performing the Non-Exclusive Functions and Duties of the Under Secretary of Commerce for Standards and Technology \& Director, National Institute of Standards and Technology 
Authority

59 This publication has been developed by NIST in accordance with its statutory responsibilities under the 60 Federal Information Security Modernization Act (FISMA) of 2014, 44 U.S.C. § 3551 et seq., Public Law 61 (P.L.) 113-283. NIST is responsible for developing information security standards and guidelines, including 62 minimum requirements for federal information systems, but such standards and guidelines shall not apply 63 to national security systems without the express approval of appropriate federal officials exercising policy authority over such systems. This guideline is consistent with the requirements of the Office of Management and Budget (OMB) Circular A-130.

Nothing in this publication should be taken to contradict the standards and guidelines made mandatory and binding on federal agencies by the Secretary of Commerce under statutory authority. Nor should these guidelines be interpreted as altering or superseding the existing authorities of the Secretary of Commerce, Director of the OMB, or any other federal official. This publication may be used by nongovernmental organizations on a voluntary basis and is not subject to copyright in the United States. Attribution would, however, be appreciated by NIST.

\section{CODEN: NSPUE2}

Certain commercial entities, equipment, or materials may be identified in this document in order to describe an experimental procedure or concept adequately. Such identification is not intended to imply recommendation or endorsement by NIST, nor is it intended to imply that the entities, materials, or equipment are necessarily the best available for the purpose.

There may be references in this publication to other publications currently under development by NIST in accordance with its assigned statutory responsibilities. The information in this publication, including concepts and methodologies, may be used by federal agencies even before the completion of such companion publications. Thus, until each publication is completed, current requirements, guidelines, and procedures, where they exist, remain operative. For planning and transition purposes, federal agencies may wish to closely follow the development of these new publications by NIST.

Organizations are encouraged to review all draft publications during public comment periods and provide feedback to NIST. Many NIST cybersecurity publications, other than the ones noted above, are available at https://csrc.nist.gov/publications.

Public comment period: February 17, 2022 - March 23, 2022

Submit comments on this publication to: applesec@nist.gov 


\section{Reports on Computer Systems Technology}

97 The Information Technology Laboratory (ITL) at the National Institute of Standards and

98 Technology (NIST) promotes the U.S. economy and public welfare by providing technical

99 leadership for the Nation's measurement and standards infrastructure. ITL develops tests, test

100 methods, reference data, proof of concept implementations, and technical analyses to advance

101 the development and productive use of information technology. ITL's responsibilities include the development of management, administrative, technical, and physical standards and guidelines for the cost-effective security and privacy of other than national security-related information in federal information systems. The Special Publication 800-series reports on ITL's research, guidelines, and outreach efforts in information system security, and its collaborative activities with industry, government, and academic organizations.

Abstract

108 The macOS Security Compliance Project (mSCP) provides resources that system administrators, security professionals, security policy authors, information security officers, and auditors can leverage to secure and assess macOS desktop and laptop system security in an automated way. This publication introduces the $\mathrm{mSCP}$ and gives an overview of the resources available from the project's GitHub site, which is continuously curated and updated to support each new release of macOS. The GitHub site provides practical, actionable recommendations in the form of secure baselines and associated rules. This publication also describes use cases for leveraging the mSCP 115 content.

\section{Keywords}

117 Apple; baseline; configuration management; endpoint device security; macOS; macOS Security 118 Compliance Project (mSCP); operating system security; security compliance.

\section{Supplemental Content}

The mSCP's GitHub site is at https://github.com/usnistgov/macos_security\#readme, and the project documentation Wiki is at https://github.com/usnistgov/macos_security/wiki.

\section{Acknowledgments}

123 The authors wish to thank Jason Blake and Blair Heiserman from NIST, Allen Golbig from Jamf, Dan Brodjieski, Gary Gapinski, and Elyse Anderson from NASA, and Jamie Richardson and Chris Stone from Apple for their contributions to the mSCP. The authors also wish to thank Stephanie Roberts from NIST for contributions to this publication. The authors appreciate Bob McSulla and Ryan Jaynes from Tenable for developing audit files based on the mSCP, testing

128 the baselines for different macOS versions, and contributing to Appendix C. The authors also 129 thank Isabel Van Wyk from NIST for editing the document. Finally, portions of this document 130 are based on content from the mSCP Wiki, so the work of all Wiki contributors is appreciated.

\section{Trademark Information}

132 All registered trademarks or trademarks belong to their respective organizations. 


\section{Call for Patent Claims}

134 This public review includes a call for information on essential patent claims (claims whose use 135 would be required for compliance with the guidance or requirements in this Information 136 Technology Laboratory (ITL) draft publication). Such guidance and/or requirements may be 137 directly stated in this ITL Publication or by reference to another publication. This call also 138 includes disclosure, where known, of the existence of pending U.S. or foreign patent applications 139 relating to this ITL draft publication and of any relevant unexpired U.S. or foreign patents.

140 ITL may require from the patent holder, or a party authorized to make assurances on its behalf, 141 in written or electronic form, either:

Such assurance shall indicate that the patent holder (or third party authorized to make assurances on its behalf) will include in any documents transferring ownership of patents subject to the assurance, provisions sufficient to ensure that the commitments in the assurance are binding on the transferee, and that the transferee will similarly include appropriate provisions in the event of

- assurance in the form of a general disclaimer to the effect that such party does not hold and does not currently intend holding any essential patent claim(s); or

- assurance that a license to such essential patent claim(s) will be made available to applicants desiring to utilize the license for the purpose of complying with the guidance or requirements in this ITL draft publication either:

○ under reasonable terms and conditions that are demonstrably free of any unfair discrimination; or

- without compensation and under reasonable terms and conditions that are demonstrably free of any unfair discrimination.

156 The assurance shall also indicate that it is intended to be binding on successors-in-interest

157 regardless of whether such provisions are included in the relevant transfer documents.

158 Such statements should be addressed to applesec@nist.gov, with the subject: "SP 800-219 Call 159 for Patent Claims." 
Executive Summary

162 The National Institute of Standards and Technology (NIST) has traditionally published secure 163 configuration guides for Apple desktop/laptop operating system versions as prose-based Special 164 Publications (SPs), such as NIST SP 800-179 Revision 1, Guide to Securing Apple macOS 10.12

165 Systems for IT Professionals: A NIST Security Configuration Checklist. In order to provide 166 security configuration guidance to organizations more quickly and in a machine-consumable 167 format, NIST has established the open-source macOS Security Compliance Project (mSCP). 168 Instead of NIST producing a prose SP guidance document for each macOS release, the mSCP 169 will continuously curate and update machine-consumable macOS guidance.

170 The mSCP seeks to simplify the macOS security development cycle by reducing the amount of 171 effort required to implement security baselines. Security baselines are groups of settings used to 172 configure a system to meet a target level or set of requirements, or to verify that a system 173 complies with requirements. The $\mathrm{mSCP}$, a collaboration among federal agencies, minimizes 174 duplicate effort that would otherwise be needed for these agencies to administer individual 175 security baselines. Additionally, the secure baseline content provided is easily extensible by 176 other parties to implement their own security requirements.

177 This document provides a high-level overview of the mSCP, its components, and some common 178 use cases. Readers seeking more detailed information on mSCP content or the content itself 179 should visit the mSCP GitHub page (https://github.com/usnistgov/macos security) and wiki 180 (https://github.com/usnistgov/macos_security/wiki).

181 Organizations using mSCP content, particularly security baseline examples, should take a risk182 based approach for selecting the appropriate settings and defining setting values that takes into 183 account the context under which the baseline will be utilized. 
185 Executive Summary .............................................................................................

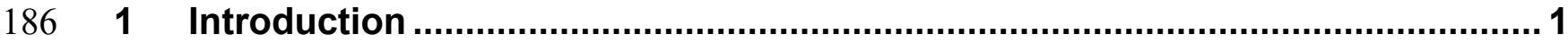

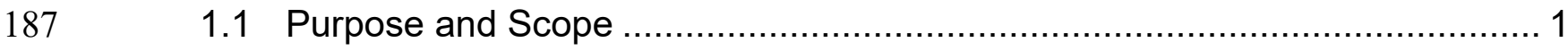

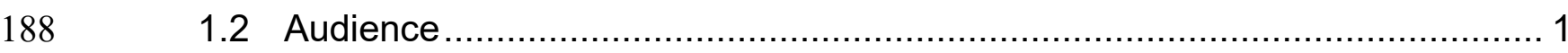

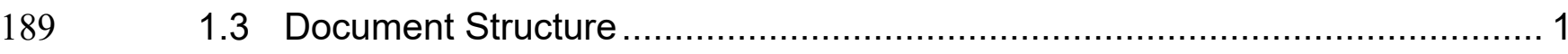

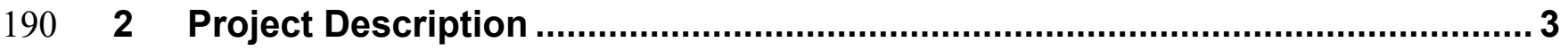

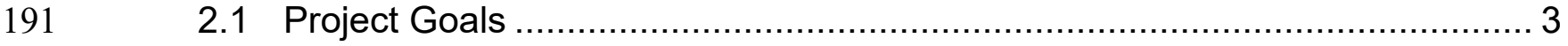

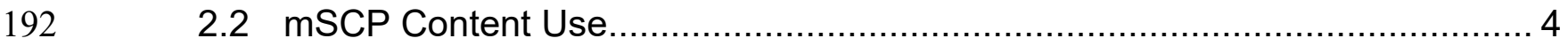

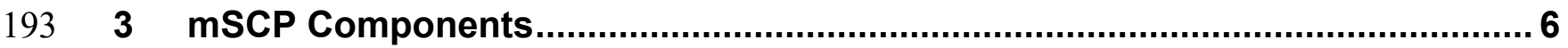

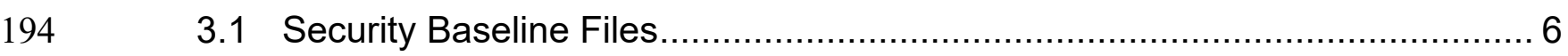

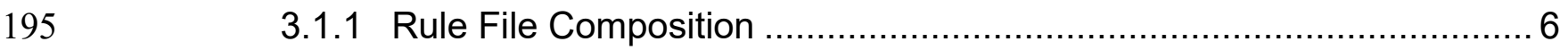

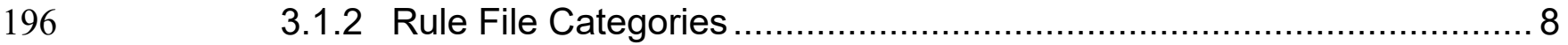

$197 \quad 3.2$ Configuration Profiles and Scripts ........................................................ 9

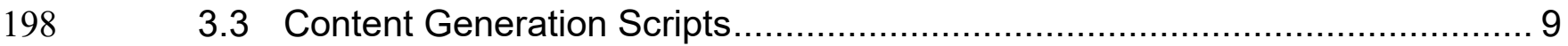

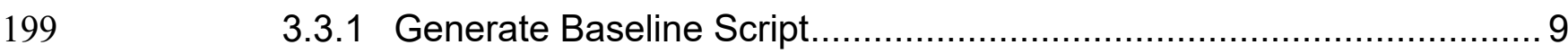

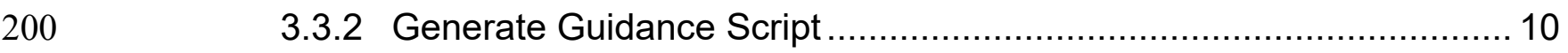

$201 \quad 3.3 .3$ macOS Security Compliance Tool............................................... 10

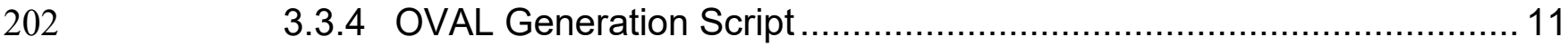

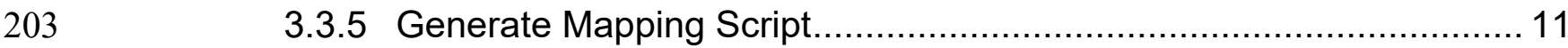

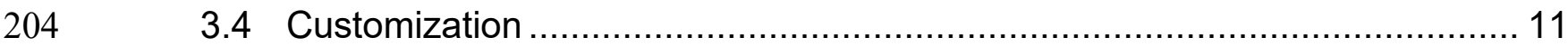

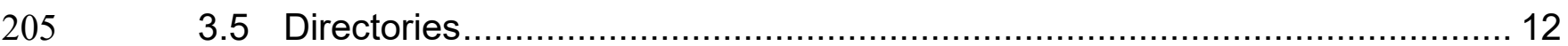

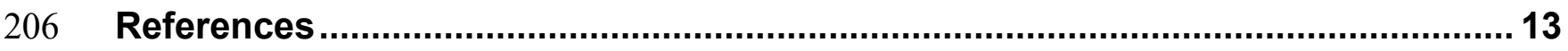

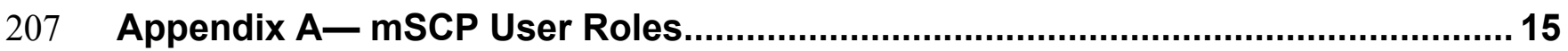

208 Appendix B- Example of mSCP Usage by a Security Professional...................... 16

209 Appendix C- Example of mSCP Usage by an Assessment Tool Vendor ............. 21

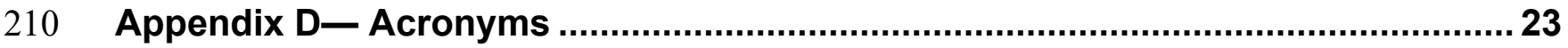




\section{Introduction}

213

214

215

216

217

218

219

220

221

222

223

224

225

226

227

The National Institute of Standards and Technology (NIST) has traditionally published secure configuration guides for Apple desktop/laptop operating system versions as prose-based Special Publications (SPs), such as NIST SP 800-179 Revision 1, Guide to Securing Apple macOS 10.12 Systems for IT Professionals: A NIST Security Configuration Checklist. NIST will no longer produce SP guidance documents for each macOS release, but instead will continuously curate and update machine-consumable guidance as part of NIST's macOS Security Compliance Project (mSCP) to keep up with each macOS release version.

The latest macOS security baseline content is maintained and updated on the mSCP GitHub page, https://github.com/usnistgov/macos security [1]. Security baselines are groups of settings used to configure a system to meet a target level or set of requirements, or to verify that a system complies with requirements. The mSCP seeks to simplify the macOS security development cycle by reducing the amount of effort required to implement security baselines. This collaboration between federal agencies minimizes duplicate effort that would otherwise be needed for these agencies to administer individual security baselines. Additionally, the secure baseline content provided is easily extensible by other parties to implement their own security requirements.

\subsection{Purpose and Scope}

The purpose of this document is to introduce the mSCP to broader audiences. This document provides a high-level overview of the $\mathrm{mSCP}$, its components, and some common use cases. It refers readers to the online project documentation for in-depth technical information and use instructions. This document is intended to be independent of macOS version releases; updates will be released as needed when there are substantial changes to the $\mathrm{mSCP}$.

The information in this document regarding the details of the mSCP GitHub site is accurate at the time of publication. Check the project wiki (https://github.com/usnistgov/macos_security/wiki) for the latest information.

The release of SP 800-219 formally deprecates NIST SP 800-179 [2] and SP 800-179 Revision 1 [3]; their applicable recommendations have already been added to corresponding $\mathrm{mSCP}$ baselines. Organizations needing to reference a NIST SP to demonstrate how they are complying with United States Government mandates for adopting secure configurations for their macOS devices may reference this SP instead of SP 800-179 or SP 800-179 Revision 1.

\subsection{Audience}

This document—and the mSCP GitHub site — are intended for system administrators, security professionals, policy authors, privacy officers, and auditors who have responsibilities involving macOS security. Additionally, vendors of device management, security, configuration assessment, and compliance tools supporting macOS may find this document and the GitHub site to be helpful.

\subsection{Document Structure}

The remaining sections and appendices of this document are as follows: 
- Section 2 provides an overview of the project, including what its goals are and how its content can be used.

- Section 3 explains the major components of the $\mathrm{mSCP}$ and provides pointers to additional information on component usage.

- The References section lists the references for the document.

- Appendix A briefly discusses how mSCP can help meet the needs of people in several roles.

- Appendix B provides examples of how a security professional might use mSCP content.

- Appendix C contains an example of how an assessment tool vendor could leverage mSCP content.

- Appendix D lists the acronyms and abbreviations used in this document. 


\section{Project Description}

262

263

264

265

266

267

268

269

270

271

272

273

274

275

276

277

278

279

280

281

282

283

284

285

286

287

288

289

290

291

292

293

294

295

296

297

The $\mathrm{mSCP}$ is an open-source project providing a programmatic approach to generating and using macOS security configuration baselines. The project's content can be used to create customized security baselines of technical security controls by leveraging a library of rules, with each rule mapped to requirements in one or more existing security standards, regulations, frameworks, etc. This approach provides versioning and consistency of the content. Unifying and standardizing macOS baseline efforts via the $\mathrm{mSCP}$ means that updating security guidance is simplified and radically accelerated, even as new versions of macOS are introduced annually.

The mSCP started in August 2019 as a collaboration among operational IT security staff from NIST, the National Aeronautics and Space Administration (NASA), the Defense Information Systems Agency (DISA), and the Department of Energy's (DOE) Los Alamos National Laboratory (LANL). ${ }^{1}$ The mSCP sought to map macOS settings to the NIST SP 800-53 Revision 4 [4] document with an extensible, modern approach to security guidance that could be used by any organization (e.g., government, enterprise, education) that needs to adhere to security compliance frameworks and policy.

As of this writing, the configuration settings represent guidance and best practices from NIST SP 800-53 Revision 5 [5], NIST SP 800-171 Revision 2 [6], the macOS DISA Security Technical Implementation Guide (STIG) [7], the Committee on National Security Systems (CNSS) Instruction (CNSSI) Number 1253 [8], and the Center for Internet Security (CIS) Critical Security Controls Version 8 [9], as well as internal organizational security guidance from NIST, NASA, and LANL.

\subsection{Project Goals}

Apple releases a new macOS version every year, and generally, agencies and organizations must wait for guidance or accept risk before deploying the new macOS version. Most agencies or organizations must create their own internal security configuration, which delays deploying the new macOS version or new hardware that only supports the new macOS version. The $\mathrm{mSCP}$ assists organizations in upgrading sooner. The technical security settings in macOS generally do not drastically change from release to release, with only a handful of new settings being introduced. By pursuing a rules-based approach, $\mathrm{mSCP}$ rules that remain applicable can be reused and incorporated into guidance for the latest macOS version. This enables quicker adoption of new security features that are not offered in prior versions of macOS.

The goals of the $\mathrm{mSCP}$ are:

- Develop recommended security baselines using a risk-based approach based on the impact of the data

- Normalize and accelerate annual adoption of the new operating system and hardware that is specific to it by providing guidance to meet the security needs of new operating systems at the earliest availability

\footnotetext{
${ }^{1}$ See https://github.com/usnistgov/macos_security\#authors for a current list of project contributors.
} 
- Reduce worldwide effort in creating annual guidance by unifying and consolidating compliance efforts into a single project

- Develop a methodology to foster collaboration between baseline authors, reducing overhead and redundancy

- Establish a unified approach for configuration and assessment of controls across multiple sources and tools

- Enable the customization of existing content and the creation of new content, including creating custom baselines in order to meet organization-specific security requirements

- Provide device management and security tool vendors, auditors, and Apple insight into customer security configuration needs

\section{2 mSCP Content Use}

mSCP content can be used by any organization to assist in setting and assessing the security configuration of macOS systems. Security baselines can be made to map to existing guidance or controls, such as those in NIST SP 800-53 Revision 5 [5], or they can be customized to meet an organization's specific needs. In mSCP terminology, a security baseline is represented as a baseline file designating rules required to meet a specific set of requirements. The $\mathrm{mSCP}$ provides a library of rules that are macOS settings. Each rule is mapped to a requirement within a security standard, framework, etc. Baseline files and rules comprise much of the mSCP's content.

The mSCP offers several example baselines, including the following, with descriptions adapted from FIPS 199 [10]:

- The SP 800-53 Revision 5 low baseline is a defined map of controls to secure a system defined as a low-impact information system. The loss of confidentiality, integrity, or availability could be expected to have a limited adverse effect on organizational operations, organizational assets, or individuals.

- The SP 800-53 Revision 5 moderate baseline is a defined map of controls to secure a system defined as a moderate-impact information system. The loss of confidentiality, integrity, or availability could be expected to have a serious adverse effect on organizational operations, organizational assets, or individuals.

- The $\boldsymbol{S P ~ 8 0 0 - 5 3 ~ R e v i s i o n ~} 5$ high baseline is a defined map of controls to secure a system defined as a high-impact information system. The loss of confidentiality, integrity, or availability could be expected to have a severe or catastrophic adverse effect on organizational operations, organizational assets, or individuals.

Organizations using any baseline example should take a risk-based approach for selecting the appropriate settings and organizationally defined values depending on the context under which the baseline will be applied. Organizations can tailor any of the baselines to include controls specific to their needs and to produce evidence of control enforcement. 
335 The mSCP provides scripts that can be used with baselines for several purposes, including the 336 following:

337 - Creating scripts and profiles for configuring macOS

338 - Generating a mapping between two security standards, regulations, frameworks, etc.

339 - Producing human-readable documentation in a variety of formats

340 - Customizing existing baselines

$341 \mathrm{mSCP}$ content can also be used to generate Security Content Automation Protocol (SCAP) 342 content for automated security compliance scans. The SCAP generated follows the SCAP 1.3 343 specification [11]. Generation of SCAP content uses an Extensible Stylesheet Language 344 Transformations (XSLT) file to create an Extensible Configuration Checklist Description Format 345 (XCCDF) checklist document with an accompanying Open Vulnerability and Assessment 346 Language (OVAL) document.

347 The XCCDF and OVAL documents are bundled into an SCAP data stream collection document 348 with accompanying files that include Common Platform Enumeration (CPE) dictionary [12] 349 information and an Open Checklist Interactive Language (OCIL) document. This creates an 350 SCAP 1.3 document that validates using the NIST SCAP Content Validation Tool ${ }^{2}$ and can be 351 used by SCAP tools on macOS. More information on SCAP content generation is available at 352 https://github.com/usnistgov/macos_security/wiki/SCAP-Content-Generation.

\footnotetext{
${ }^{2}$ https://csrc.nist.gov/projects/security-content-automation-protocol/scap-releases/scap-1-3
} 


\section{3 mSCP Components}

354

355

356

357

This section provides an overview of several components of the mSCP: security baseline files, configuration profiles and scripts, content generation scripts, customization capabilities, and directories. More information about all of these is available from the GitHub wiki at https://github.com/usnistgov/macos security/wiki.

\subsection{Security Baseline Files}

In the $\mathrm{mSCP}$, a security baseline is defined in a Yet Another Markup Language (YAML) file. A YAML file is a human-readable file format commonly used by configuration files where data is stored and/or transmitted. A baseline YAML file consists of the following required fields. The code immediately below this list provides a partial example of a YAML file that illustrates the use of these fields (with field names bolded).

- title - a human-readable name for the baseline

- description - a short description of the baseline, including its use case and target operating system (OS) version

- authors - developers of the baseline

- profile - the security content portion of the baseline

○ section - a keyword for organizing settings

- rules - the names of the rule files that are a part of this baseline

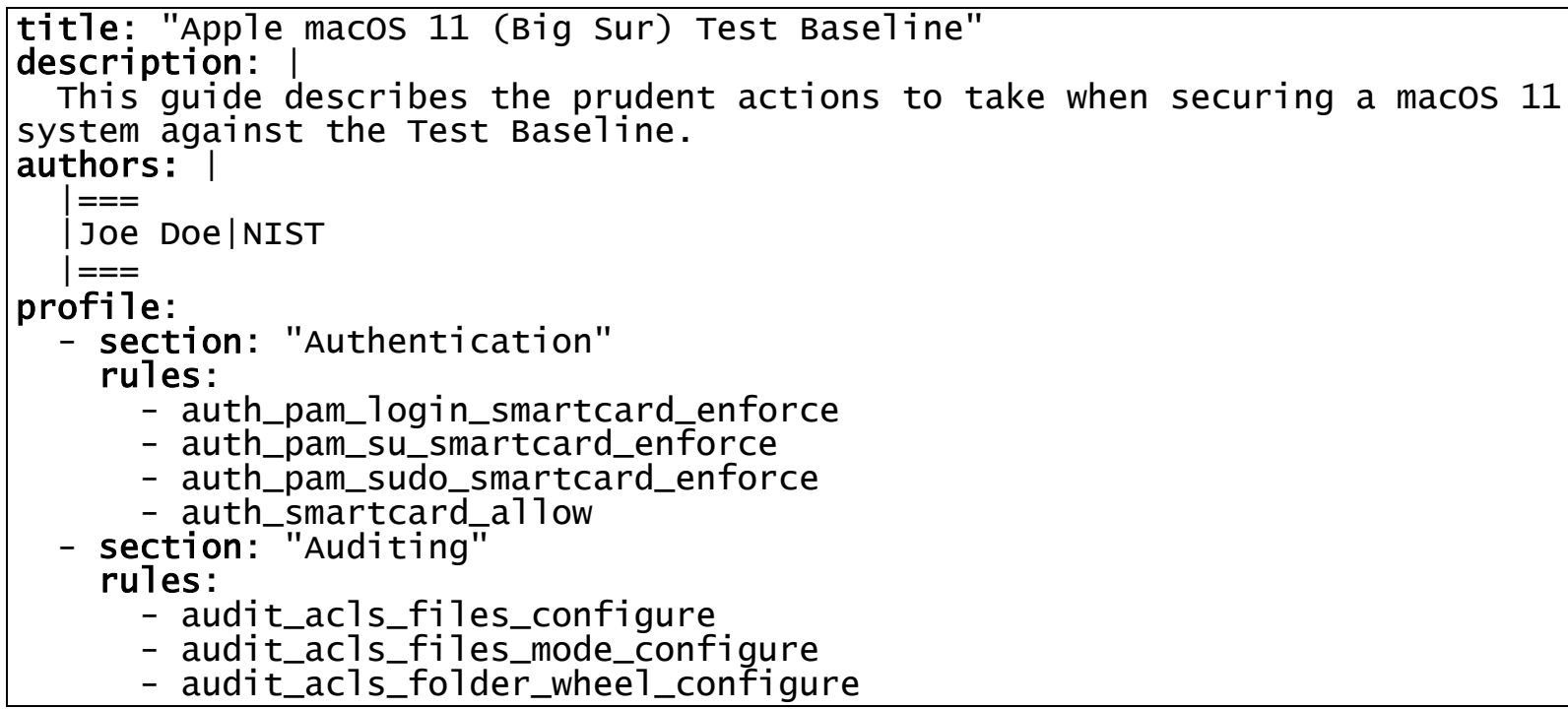

\subsubsection{Rule File Composition}

A YAML rule file is broken down into the following subsections. The code immediately below this list provides a notional example of a YAML rule file (with field names bolded). This example is from the Rules section of the mSCP wiki (https://github.com/usnistgov/macos_security/wiki/Rules). 
396 - id - the name of the rule file, excluding the .yam7 file extension

397 - title - a human-readable rule title

398 - discussion - a short description of the rule and its use case

399 - check - the check to assess the system for the specified rule; typically this is shell code

400 - result - the expected result of running the check

401 - fix - the necessary fix in case the check fails; if [source, bash] is included, the fix will be included in the configuration script

403 - references - references, including identifiers and mappings such as security frameworks, guidance, and controls; the references always include a Common Configuration Enumeration (CCE) identifier, which is assigned to this rule file and can be found in the official repository of NIST CCEs [13]

- macos - the validated macOS version for this rule

- tags - modifiable keywords for categorizing and identifying related rules

- severity - the severity level specified in the DISA STIG, if applicable

- mobileconfig - if true, this rule will be used to generate configuration profile content

- mobileconfig_info-if mobileconfig is set to true, this field specifies the information required to produce configuration profile content 


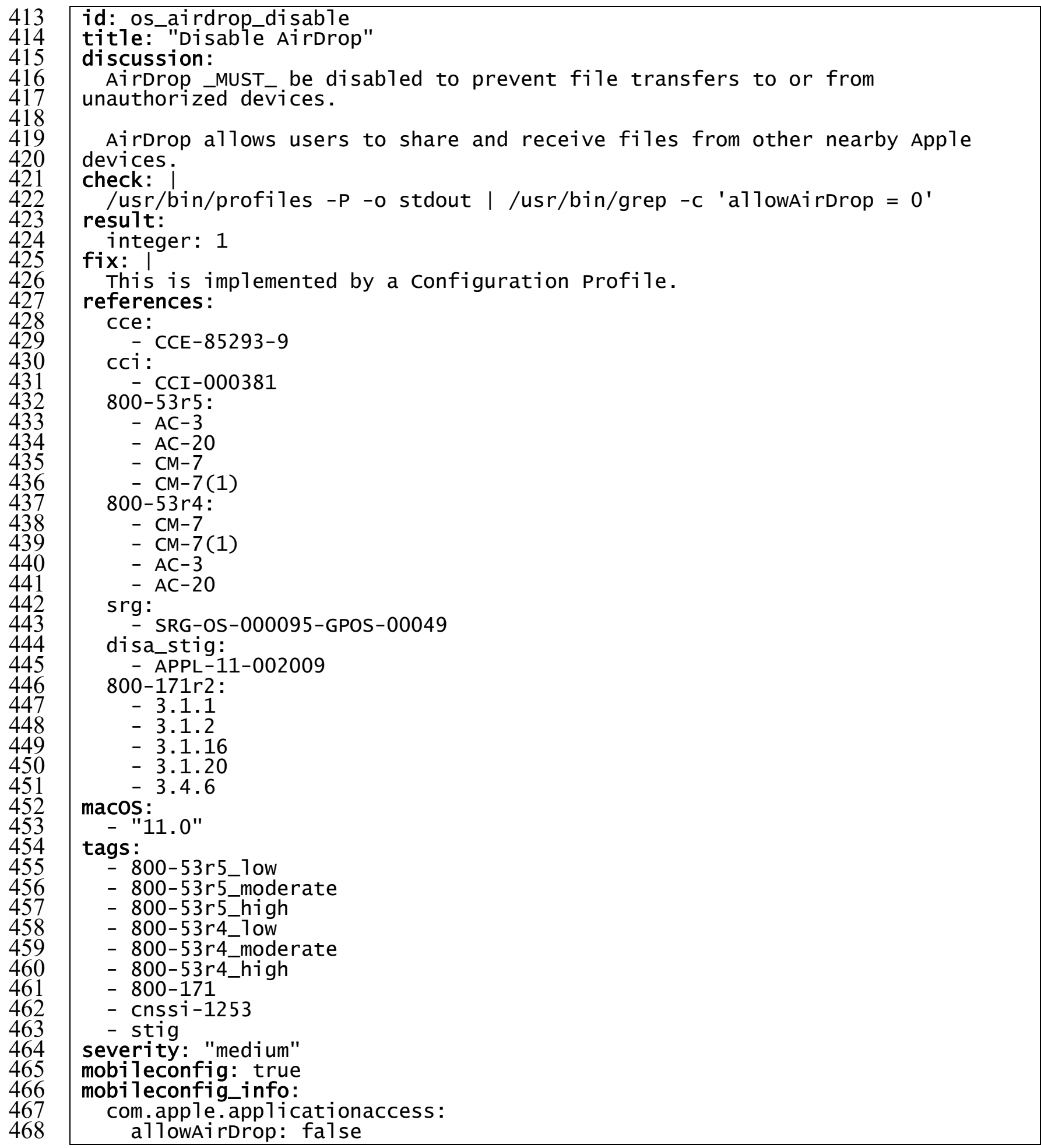

\subsubsection{Rule File Categories}

470 The mSCP organizes YAML files in the rules directory into the following subdirectories, each 471 corresponding to a category of settings: 
- $\quad$ audit - OpenBSM

- $\quad$ auth - smartcard authentication

- icloud - Apple's iCloud/Apple ID service

- os - settings that do not fit into the other categories

- $\quad$ pwpolicy - password policy

- sysprefs - settings controlled within the System Preferences application

The rules directory also includes a supplementa1 subdirectory, which contains additional information that supports the guidance provided by the baselines. Supplemental content contains information for rules that are not part of an existing baseline but could be beneficial for certain use cases. Supplemental content may not have mappings and may or may not contain the YAML rule file check and fix sections mentioned in Section 3.1.1. Supplemental content can be added to enhance baselines where organizational requirements are different than the system baseline requirements.

\subsection{Configuration Profiles and Scripts}

When an mSCP YAML file is processed, it yields a configuration script and/or configuration profile (mobi leconfig file) as outputs. Both are used to apply configuration settings to a system.

A configuration profile is an Extensible Markup Language (XML) formatted file with a mobi 1 econfig extension containing a configuration payload. macOS can automatically configure itself based on a mobi leconfig file's contents upon execution. Configuration profiles offer a convenient, Apple-supported mechanism for applying security settings to a macOS environment. Additionally, they can be cryptographically signed to ensure integrity and authenticity. These factors make configuration profiles the preferred vehicle for configuration delivery. However, mobileconfig files cannot modify all macOS settings, so a configuration script is needed for those that are not supported.

496 A configuration script is a shell script that manipulates operating system files directly. The script content is derived from all YAML rule files that have a mobi leconfig value of false and belong to the specified baseline. The YAML rule file must contain the fix section in order to generate its corresponding configuration script entry.

\subsection{Content Generation Scripts}

501 The mSCP provides several types of scripts for generating baselines, human-readable guidance, 502 baseline compliance checkers, and other types of content. Each script is described below.

\subsubsection{Generate Baseline Script}

504 The generate_base 1 ine.py script compiles a list of security rules into a single baseline YAML 505 file. It can be used to modify an existing security baseline or create a new one. See

506 https://github.com/usnistgov/macos_security/wiki/Scripts\#generate_baselinepy for additional 507 information. 


\subsubsection{Generate Guidance Script}

509 The generate_guidance.py script can produce human-readable guidance as well as generate 510 the macOS Security Compliance Tool in the form of a $\mathrm{Z}$ shell script.

511 The generate_guidance.py script takes a baseline file and produces a human-readable guide in 512 the format of documentation from information available in the YAML rules files. The 513 documentation can be in any of several formats. The script always generates an Asciidoc file. 514 AsciiDoc (.adoc) is a plain text format that uses markup conventions for traditional document 515 formatting and organization. AsciiDoc files are easily transformable into many other formats via 516 the generate_gui dance. py script, including Hypertext Markup Language (HTML), PDF, and 517 Excel. The Excel format is particularly useful for quickly viewing all the rules of a baseline, and 518 it contains all the data in the YAML rules files.

519 The generate_guidance.py script can also create configuration profiles (mobi leconfig files) and a compliance script. Using the $-\mathrm{s}$ argument, the generate_guidance.py script will generate an org. \{baseline\}. audit.plist file and another script, the macOS Security Compliance Tool that can check and remediate compliance settings. The audit.p1 ist file can be used to set an exemption to organizational rules for approved users so that compliance checks can succeed without findings. To create an exemption for a rule, the exempt field should be set to true and an exempt_reason should be added.

526 See https://github.com/usnistgov/macos security/wiki/Scripts\#generate guidancepy-script for more information on the generate_guidance. py script.

\subsection{3 macOS Security Compliance Tool}

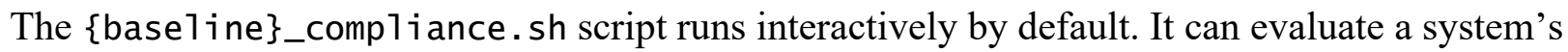
conformance to a baseline or remediate any incorrectly configured settings. Alternatively, the script can autonomously assess a system with the -check argument or automatically remediate

532 any possible settings with - fix.

533 The lines below provide an example of the results of running the script.

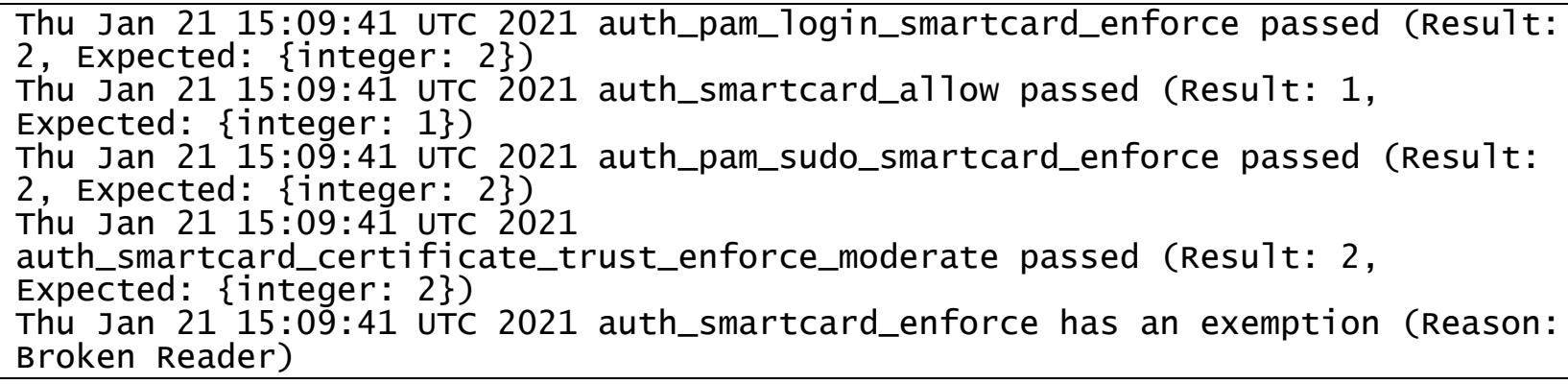

545 For more information on the macOS Security Compliance Tool script, see

546 https://github.com/usnistgov/macos_security/wiki/Compliance-Script. 


\subsubsection{OVAL Generation Script}

548 The OVAL generation script, yam 1-to-ova1 . py, takes a baseline YAML file and generates

549 OVAL checks for any rule file where possible. Note that this script does not recognize any

550 custom settings. For more information, see

551 https://github.com/usnistgov/macos_security/wiki/Scripts\#yaml-to-ovalpy.

\subsubsection{Generate Mapping Script}

The generate_mapping.py script allows for the quick creation of custom rules and baselines for a compliance framework not published by the mSCP. The script requires a user-created commaseparated values (CSV) file containing control identifiers that maps to a new framework (CSV column 1) from another already defined by the project (CSV column 2). By default, the script is designed to map a framework to the NIST SP 800-53r5 [5] set of controls. Adding the $-f$ argument allows for mapping to another supported framework. See https://github.com/usnistgov/macos_security/wiki/Generate-Mapping for more information on the generate_mapping.py script.

\subsection{Customization}

562 Customization allows organizations to generate their own customized content outside of that provided by the project. Additionally, it allows them to add content for internal-only controls, which are not suitable for inclusion in a global baseline. Customization primarily takes place within the custom folder. Here are examples of customization supported by mSCP:

- Baselines: A base1 ine folder can be included within the custom folder to create customized baselines that fit an organization's needs. These baseline files may include rule, section, and template customization (discussed below).

- Rules: Existing rules can have their setting values overridden via the custom folder instead of modifying the mSCP-supplied rule file. New rules can be created and added to existing baselines or to user-defined baselines. Organizations can create their own discussions, checks, results, fixes, and mappings of rules to security frameworks not included in the project. In order to override an existing rule, the custom rule file name must match an existing rule so the generate_guidance. py script will pick up the new values. New rules not included in mSCP must be listed in the baseline YAML file specified when running generate_guidance.py. Additional information on custom rules can be found in an article written by mSCP contributor Allen Golbig [14].

- Sections: Custom sections can be used to organize existing or custom YAML rule files. Sections defined in the custom folder must be included in a baseline YAML file in order to be used by generate_guidance.py.

- Templates: Custom templates can be used to define new template structures for the project and affect the organization and appearance of generated documentation. The template files must match the name of an existing template and will override that template when running generate_guidance.py.

- Logos: An organization can include a custom logo when running the generate_guidance. py script by using the -1 argument to point to an image file. 


\subsection{Directories}

$588 \mathrm{mSCP}$ releases available at https://github.com/usnistgov/macos security/releases include the 589 following directories:

590 - base1ines - contains the defined YAML baseline files

591 - bui1d - holds scripts, documents, and configuration profiles generated by running scripts

- custom - used for creating customized baselines, rules, sections, or templates to meet an organization's requirements

594 includes - contains YAML-based libraries required for running the scripts

595 - rules - contains YAML rule files, with one rule per file

596 - SCAP - contains the required files for generating SCAP content

597 - scripts - contains the content generation scripts, along with their required files

- sections - defines the sections that correlate to the directories in the rules folder; each section has its own YAML file containing the section name and description as it will appear in the generated guide, which is human-readable documentation

- $\quad$ templates - includes AsciiDoc templates for generating an AsciiDoc guide 
References

[1] macOS Security Compliance Project (2022) macOS Security Compliance Project. Available at https://github.com/usnistgov/macos security

[2] Trapnell M, Scarfone KA, Trapnell E, Badger ML, Souppaya MP, Yaga DJ (2016) Guide to Securing Apple OS X 10.10 Systems for IT Professionals: A NIST Security Configuration Checklist. (National Institute of Standards and Technology, Gaithersburg, MD), NIST Special Publication (SP) 800-179. https://doi.org/10.6028/NIST.SP.800-179

[3] Trapnell M, Scarfone KA, Trapnell E, Badger ML, Souppaya MP, Yaga DJ (2018) Guide to Securing Apple macOS 10.12 Systems for IT Professionals: A NIST Security Configuration Checklist. (National Institute of Standards and Technology, Gaithersburg, MD), Draft NIST Special Publication (SP) 800-179, Rev. 1. Available at https://csrc.nist.gov/publications/detail/sp/800-179/rev-1/draft

[4] Joint Task Force Transformation Initiative (2013) Security and Privacy Controls for Federal Information Systems and Organizations. (National Institute of Standards and Technology, Gaithersburg, MD), NIST Special Publication (SP) 800-53, Rev. 4, Includes updates as of January 22, 2015. https://doi.org/10.6028/NIST.SP.800-53r4

[5] Joint Task Force (2020) Security and Privacy Controls for Information Systems and Organizations. (National Institute of Standards and Technology, Gaithersburg, MD), NIST Special Publication (SP) 800-53, Rev. 5. Includes updates as of December 10, 2020. https://doi.org/10.6028/NIST.SP.800-53r5

[6] Ross R, Pillitteri V, Dempsey K, Riddle M, Guissanie G (2020) Protecting Controlled Unclassified Information in Nonfederal Systems and Organizations. (National Institute of Standards and Technology, Gaithersburg, MD), NIST Special Publication (SP) 800171, Rev. 2, Includes updates as of January 28, 2021. https://doi.org/10.6028/NIST.SP.800-171r2

[7] Department of Defense (2021) DISA STIG for macOS. Available at https://public.cyber.mil/stigs/

[8] Committee on National Security Systems (2014) Security Categorization and Control Selection for National Security Systems. (National Security Agency, Ft. Meade, MD), Committee on National Security Systems Instruction (CNSSI) No. 1253. Available at https://www.cnss.gov/CNSS/issuances/Instructions.cfm

[9] Center for Internet Security (2021) CIS Critical Security Controls Version 8. Available at https://www.cisecurity.org/controls/v8/

[10] National Institute of Standards and Technology (2004) Standards for Security Categorization of Federal Information and Information Systems. (U.S. Department of Commerce, Washington, DC), Federal Information Processing Standards Publication (FIPS) 199. https://doi.org/10.6028/NIST.FIPS.199

[11] Waltermire DA, Quinn SD, Booth H, III, Scarfone KA, Prisaca D (2018) The Technical Specification for the Security Content Automation Protocol (SCAP): SCAP Version 1.3. (National Institute of Standards and Technology, Gaithersburg, MD), 
NIST Special Publication (SP) 800-126, Rev. 3. https://doi.org/10.6028/NIST.SP.800$126 \mathrm{r} 3$

[12] National Institute of Standards and Technology (2021). Official Common Platform Enumeration (CPE) dictionary. Available at https://nvd.nist.gov/products/cpe

[13] National Institute of Standards and Technology (2021) CCE Platform Listing. Available at https://ncp.nist.gov/cce

[14] Golbig A (2021) Getting to Know: macOS Security Compliance Project-Part 2. Available at https://golbiga.medium.com/getting-to-know-macos-security-complianceproject-part-2-24131b60cdfb 
Appendix A-mSCP User Roles

604 The mSCP was designed to meet the needs of different security roles. These perspectives are

605 briefly examined below.

606 Security policy authors define the policies for their organizations. The customization and ease 607 of extensibility offered by the mSCP facilitate new content creation. Policy authors will need to 608 familiarize themselves with the YAML rule file format described in Section 3.1.1. Of particular 609 interest is the ability to map rules directly to references. Additionally, the generate mapping 610 script (Section 3.3.5) enhances portability between compliance frameworks.

611 System administrators and security professionals are responsible for configuring the systems 612 under their purview. They implement the guidance issued by security policy authors. As such, 613 configuration tools such as the macOS Security Compliance Tool's (Section 3.3.3) automatic 614 remediation mode are of interest. Additionally, security professionals may wish to generate 615 baselines (Section 3.3.1), guidance (Section 3.3.2), and the macOS Security Compliance Tool 616 (Section 3.3.3).

617 Auditors approach macOS security compliance from a validator perspective, seeking proof that 618 a system is configured in the required way. They are more interested in system setting 619 documentation and compliance evidence than technical tools such as configuration scripts. Both 620 of these needs can be met by mSCP tools. The generate guidance script (Section 3.3.2) provides 621 the necessary documentation in a variety of formats including HTML, PDF, and Excel. The 622 macOS Security Compliance Tool (Section 3.3.3) assesses a system and produces a log of the 623 results. Additionally, some auditors may be interested in examining YAML rule content directly 624 (Section 3.1.1).

625 Information security officers have a variety of goals but are ultimately responsible for ensuring 626 that systems are configured according to their organizational requirements. To accomplish this, 627 they need policy documentation (Section 3.3.2) and the results of compliance scans (Section 628 3.3.3). Information security officers may also be responsible for reviewing the security rules 629 proposed by the policy authors. If this is the case, they may be interested in YAML rule file 630 components (Section 3.1.1).

631 Vendors of device management, security, configuration assessment, and compliance tools 632 can produce a series of audit files based on mSCP content to support different macOS versions 633 and associated security baselines. These audit files are maintained, tested, published, and 634 supported by the tool vendors. Tool customers can download and import the content into the tool 635 to assess the state of their system against a particular baseline in an automated way.

636 Specific audit files of the mSCP by tool vendors are described on the project wiki page. This 637 content will be updated as contributing tool vendors develop new audit content. 
Appendix B-Example of mSCP Usage by a Security Professional

639 This appendix provides examples of how a security professional might use mSCP content.

640 People in other roles might perform some of the same actions. The examples illustrated below

641 were accurate at the time of publication, but please see the mSCP wiki at

642 https://github.com/usnistgov/macos_security/wiki for up-to-date usage guidance. Note that the

$643 \mathrm{mSCP}$ scripts are not meant to replace enterprise-class configuration and management tools.

644 Configurations should be tested on development systems before being deployed on end users'

645 systems.

\section{Preparing to use $\mathbf{m S C P}$}

647 All project components are available from the mSCP GitHub page [1] by navigating to 648 Releases and downloading the latest source code revision for the desired macOS version.

649 Alternatively, the project source code can be downloaded via git, as the example below 650 illustrates.

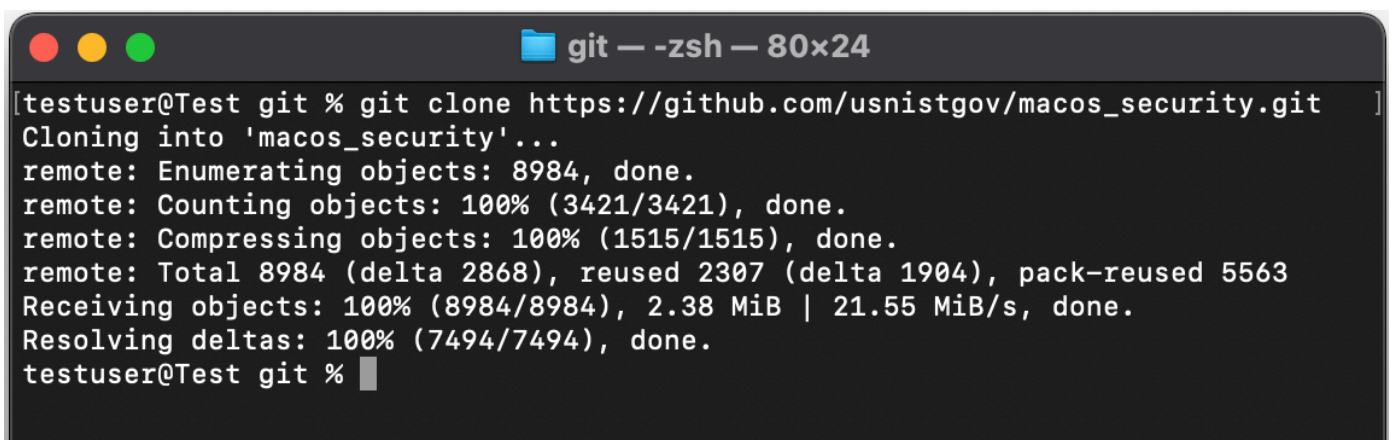

$652 \mathrm{mSCP}$ components rely on prerequisite software listed at

653 https://github.com/usnistgov/macos_security/wiki/Getting-Started, so any missing software will

654 need to be installed.

\section{Changing code branches and generating a baseline}

656 After obtaining a copy of the source code, change directory to the $\mathrm{mSCP}$ git folder, 657 macos_security.

659 Next, select the appropriate code branch that corresponds to the target OS version. Then choose a

660 baseline and use the generate_base1 ine.py script to create a baseline YAML file. The

661 example below illustrates these steps for the NIST SP 800-53 Revision 5 moderate baseline for 662 macOS Big Sur. 
testuser@Test macos_security \% git checkout big_sur

Branch 'big_sur' set up to track remote branch 'big_sur' from 'origin'.

Switched to a new branch 'big_sur'

[testuser@Test macos_security \% ./scripts/generate_baseline.py -k 800-53r5_moderate

testuser@Test macos_security $\%$

\section{Creating the macOS Security Compliance Tool and configuration profiles}

665 Using the generate_guidance.py script, create the macOS Security Compliance Tool and

666 configuration profiles. The example below illustrates this, continuing from the previous example.

macos_security $--\mathrm{zsh}-102 \times 24$

[testuser@Test macos_security \% ./scripts/generate_guidance.py -s -p baselines/800-53r5_moderate.yam]

Profile YAML: baselines/800-53r5_moderate.yaml

Output path: /Users/testuser/git/temp/macos_security/build/800-53r5_moderate/800-53r5_moderate.adoc

Generating configuration profiles...

Configuration profile written to /Users/testuser/git/temp/macos_security/build/800-53r5_moderate/mobil econfigs/unsigned/com.apple.security.smartcard.mobileconfig

Settings plist written to /Users/testuser/git/temp/macos_security/build/800-53r5_moderate/mobileconfig s/preferences/com.apple.security.smartcard.plist

Configuration profile written to /Users/testuser/git/temp/macos_security/build/800-53r5_moderate/mobil econfigs/unsigned/com.apple.applicationaccess.mobileconfig

Settings plist written to /Users/testuser/git/temp/macos_security/build/800-53r5_moderate/mobileconfig s/preferences/com.apple.applicationaccess.plist

Configuration profile written to /Users/testuser/git/temp/macos_security/build/800-53r5_moderate/mobil econfigs/unsigned/com.apple.SetupAssistant.managed.mobileconfig

Settings plist written to /Users/testuser/git/temp/macos_security/build/800-53r5_moderate/mobileconfig s/preferences/com.apple.SetupAssistant.managed.plist

Configuration profile written to /Users/testuser/git/temp/macos_security/build/800-53r5_moderate/mobil econfigs/unsigned/com.apple.mDNSResponder.mobileconfig

Settings plist written to /Users/testuser/git/temp/macos_security/build/800-53r5_moderate/mobileconfig s/preferences/com.apple.mDNSResponder.plist

Configuration profile written to /Users/testuser/git/temp/macos_security/build/800-53r5_moderate/mobil econfigs/unsigned/com.apple.applicationaccess.new.mobileconfig

Settings plist written to /Users/testuser/git/temp/macos_security/build/800-53r5_moderate/mobileconfig s/preferences/com.apple.applicationaccess.new.plist

\section{Running a compliance scan}

669 As the example below shows, the macOS Security Compliance Tool is typically run with 670 administrator privileges so that it can access all the settings.

672 The example below shows the main menu presented by the macOS Security Compliance Tool. 


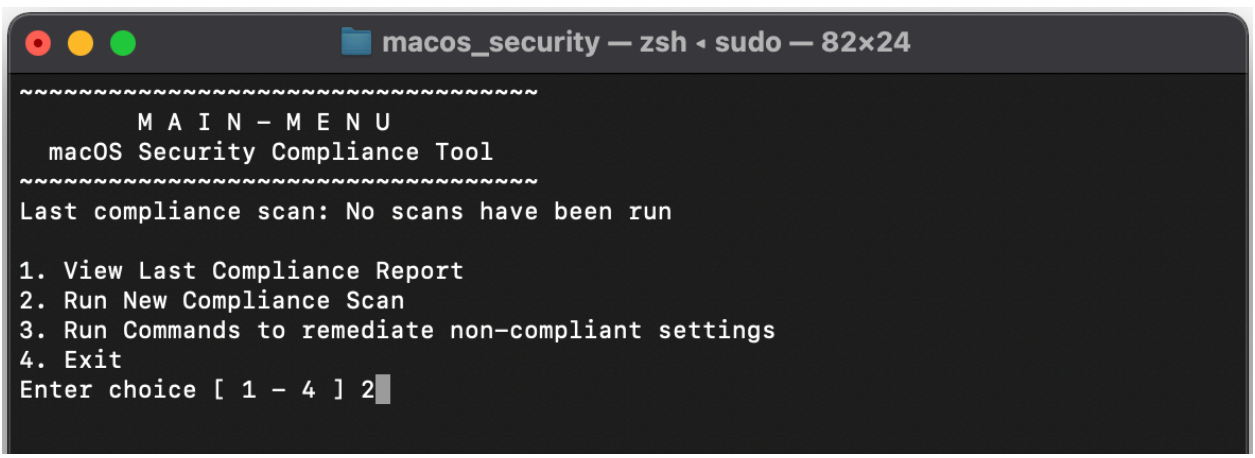

674 Selecting option 2, "Run New Compliance Scan," from the main menu launches the scan. The 675 example below shows output from the scan, which in this case reflects numerous rule failures, 676 each indicating a deviation from the expected configuration.

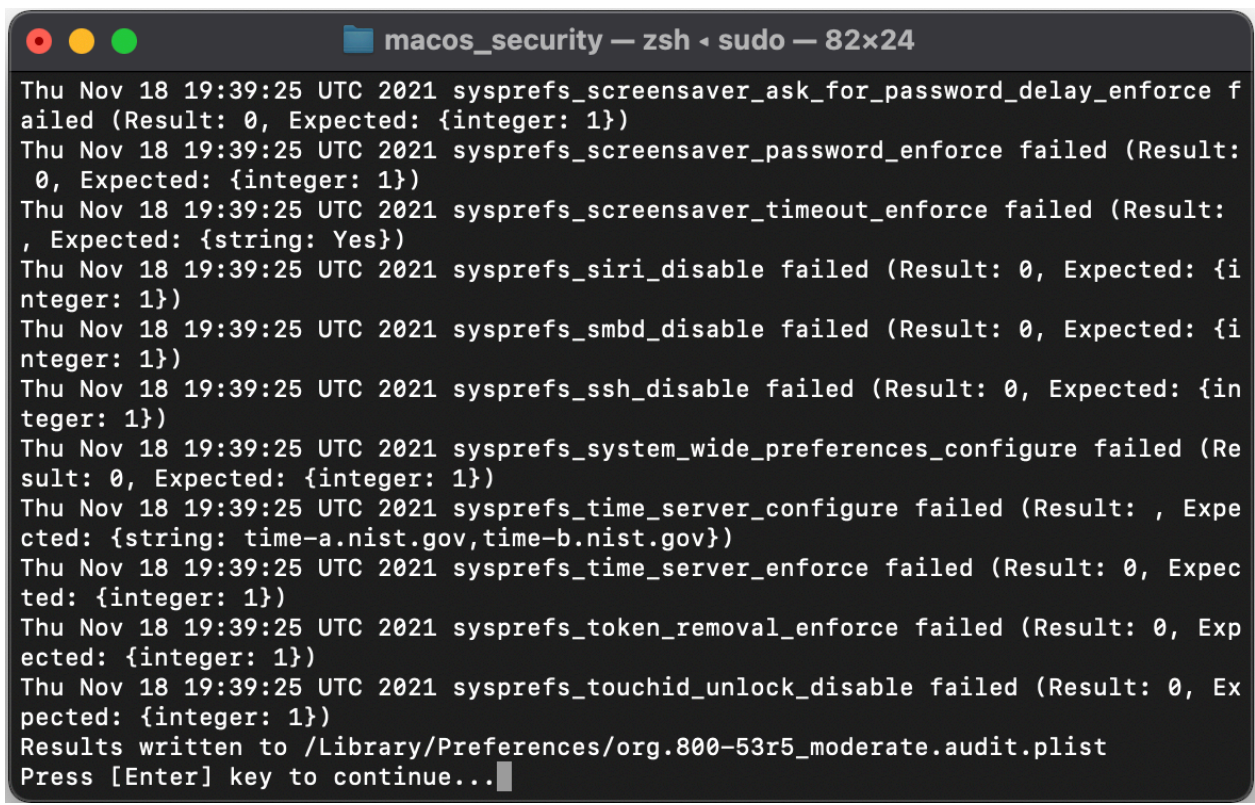

678 Selecting option 1, "View Last Compliance Report," from the main menu displays a summary of 679 the compliance report results. The example below depicts results indicating that 30 tests passed 680 and 108 tests failed, for an overall score of $21.74 \%$ compliant. 


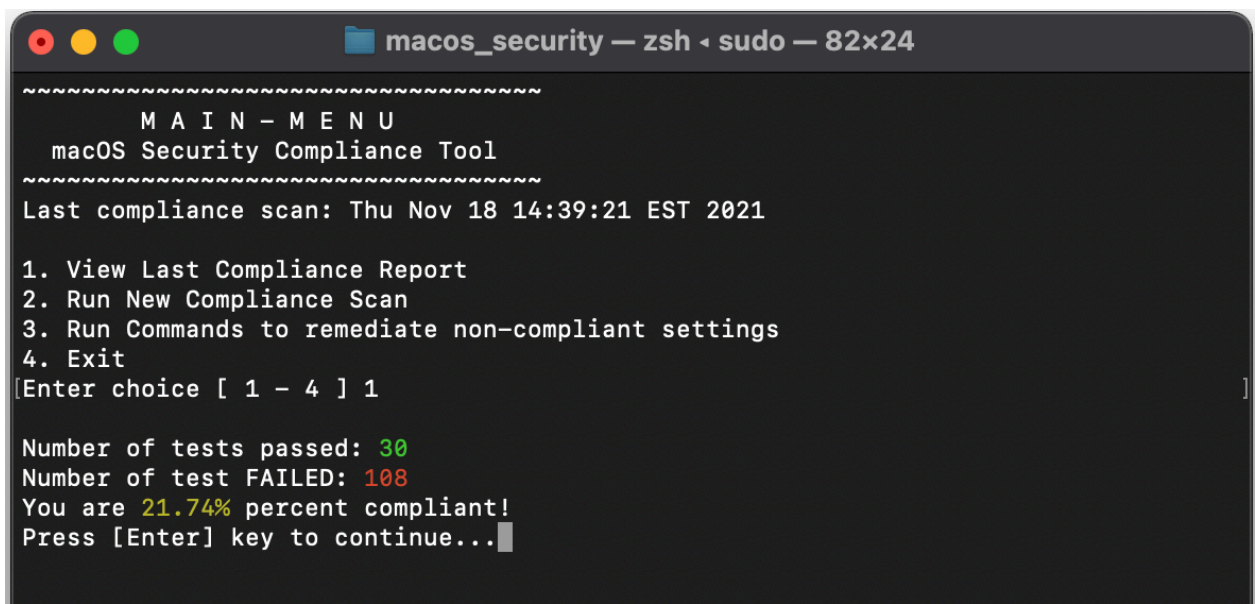

\section{Fixing non-compliant settings}

683 Selecting option 3, "Run Commands to remediate non-compliant settings," begins the process of 684 fixing non-compliant settings discovered during a previous compliance scan. The example below 685 illustrates the disclaimer to be reviewed and accepted before fixes are initiated. This disclaimer 686 indicates the potential risk in applying fixes.

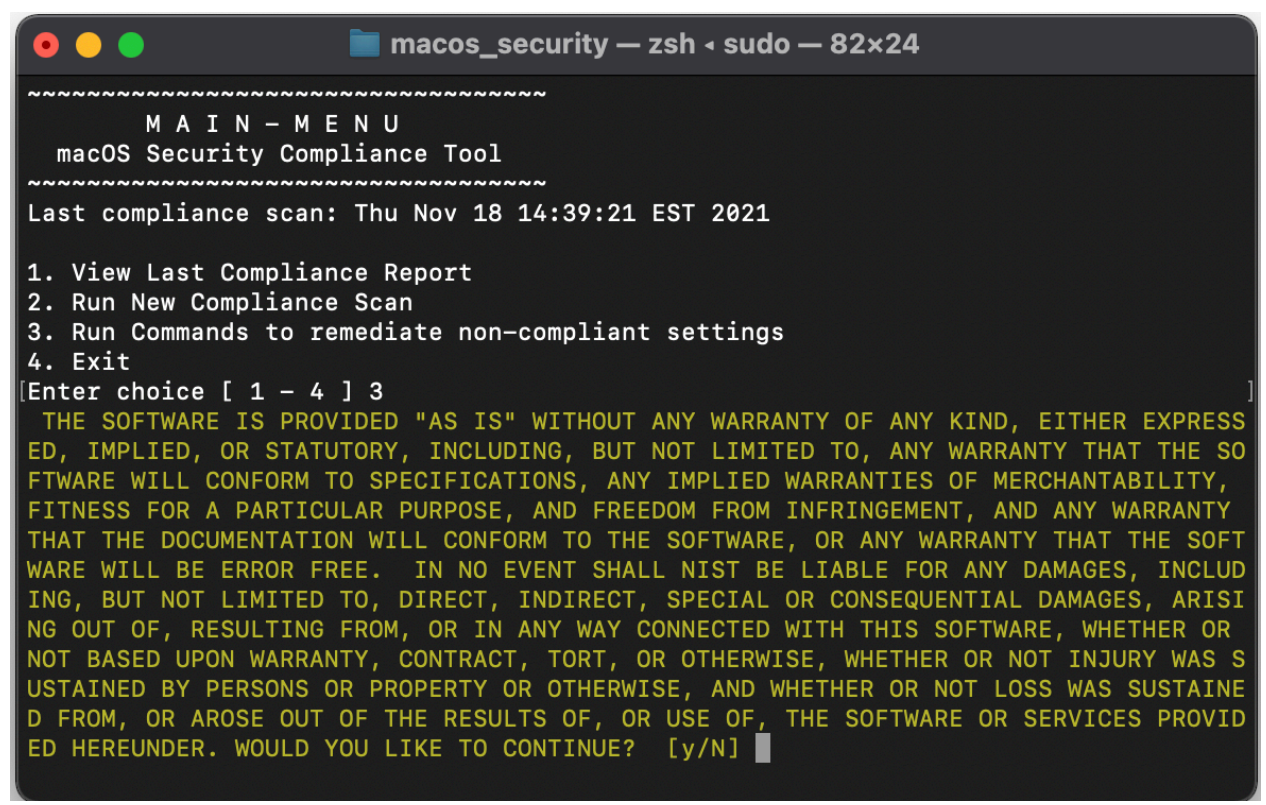

688 After the disclaimer statement is accepted, the fixes are applied to the system, as the example

689 below illustrates. 


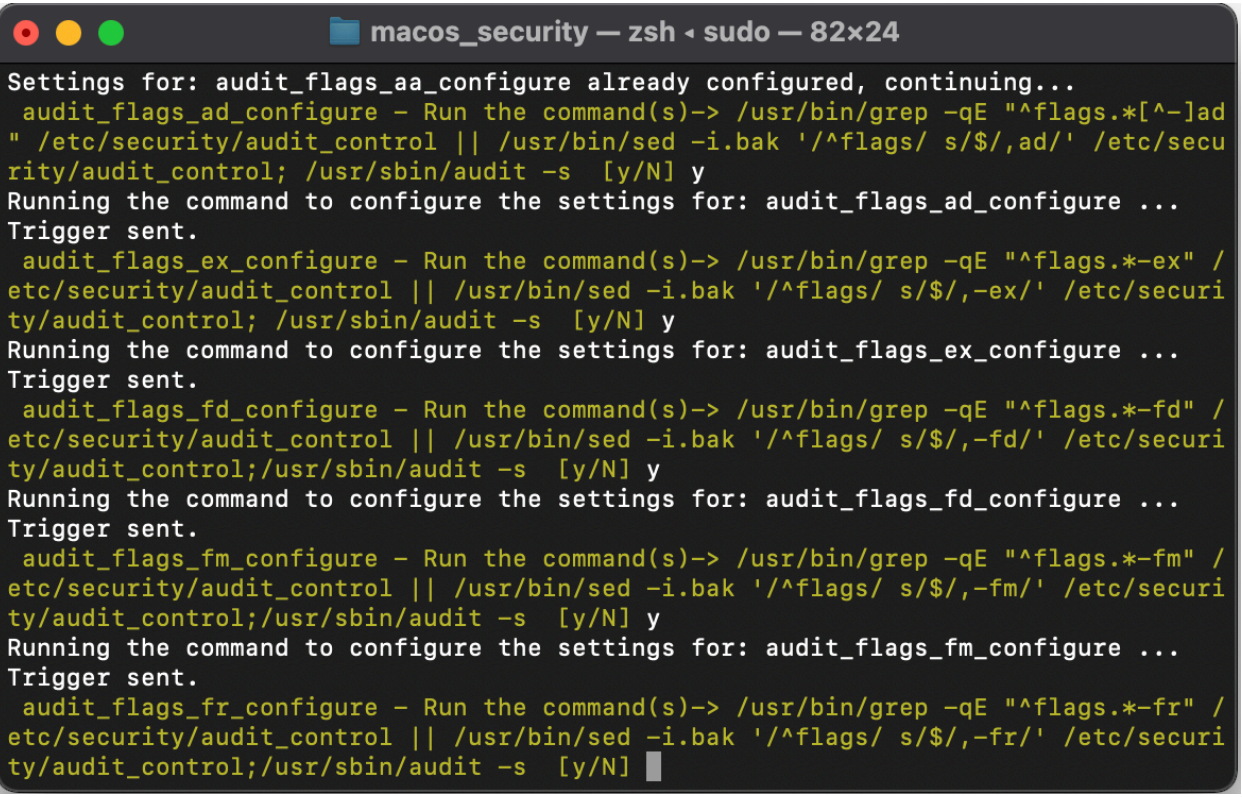


Appendix C-Example of mSCP Usage by an Assessment Tool Vendor

692 This appendix provides an example of how an assessment tool vendor converted mSCP content

693 to their tool's proprietary format so their tool could perform compliance checks against mSCP

694 baselines and rules. Refer to the mSCP GitHub wiki page for the most current list of tool vendors

695 and associated content that will support the mSCP baselines.

696 This example is for Tenable, Inc. They automated the conversion of mSCP YAML rules into

697 their audit format using Python and YAML libraries. Programmatically approaching this

698 conversion allows for faster future releases and greater consistency, and it also maintains the

699 integrity of the source content. Because the YAML content is all command-driven, it is

700 converted to Tenable's CMD_EXEC check type for use with the Unix plugin. The YAML rules

701 have a "tags" section that was used to create unique audit profiles related to common

702 frameworks. An example of these profiles can be seen in the audit file naming convention:

- NIST_mac0S_Big_Sur_800-171_v1.4.0.audit

- NIST_macos_catalina_800-53r5_high_v1.5.0.audit

706 https://community.tenable.com/s/feed/0D53a00008E0hgYCAR for more details.

707 The following example shows a YAML-to-audit-check conversion. The content has been 708 condensed and abbreviated for the purposes of comparison:

MSCP YAML

title: "Limit SSHD to FIPS 140 validated Ciphers"

discussion: |

If SSHD is enabled then it _MUST_ be configured to limit the ciphers to algorithms that are FIPS 140 validated.

FIPS 140-2 is the current standard for validating that mechanisms used to access cryptographic modules utilize authentication that meet federal requi rements.

operating systems utilizing encryption_MUST_ use FIPS validated mechanisms for authenticating to cryptographic modules.

NOTE: /etc/ssh/sshd_config will be automatically modified to its original state following any update or major upgrade to the operating system.

check: I

/usr/bin/grep -c "^Ciphers aes256-ctr,aes192-ctr,aes128-ctr"

/etc/ssh/sshd_config

result:

integer: 1

Tenable Audit Check

description : "Big Sur - Limit SSHD to FIPS 140 validated ciphers"

info : "If SSHD is enabled then it_MUST_ be configured to limit the ciphers to algorithms that are FIPS 140 validated.

FIPS 140-2 is the current standard for validating that mechanisms used to access cryptographic modules utilize authentication that meet federal

738 requirements. 
operating systems utilizing encryption_MUST_ use FIPS validated mechanisms for authenticating to cryptographic modules.

NOTE: /etc/ssh/sshd_config wi11 be automatically modified to its original

state following any update or major upgrade to the operating system."

cmd : "/usr/bin/grep -c \"^Ciphers aes256-ctr,aes192-ctr, aes128-

$<$ /custom_item> 
Appendix D-Acronyms

749 Selected acronyms and abbreviations used in this paper are defined below.

CCE Common Configuration Enumeration

CIS Center for Internet Security

CNSS Committee on National Security Systems

CNSSI Committee on National Security Systems Instruction

CSV Comma-Separated Values

DISA Defense Information Systems Agency

DOE Department of Energy

FIPS Federal Information Processing Standards

FISMA Federal Information Security Modernization Act

FOIA Freedom of Information Act

GUI Graphical User Interface

HTML Hypertext Markup Language

IT Information Technology

ITL Information Technology Laboratory

LANL Los Alamos National Laboratory

mSCP macOS Security Compliance Project

NASA National Aeronautics and Space Administration

NIST National Institute of Standards and Technology

OCIL Open Checklist Interactive Language

OS Operating System

OVAL Open Vulnerability and Assessment Language

SCAP Security Content Automation Protocol

SP Special Publication

STIG Security Technical Implementation Guide

XCCDF Extensible Configuration Checklist Description Format

XML Extensible Markup Language

XSLT Extensible Stylesheet Language Transformations

YAML Yet Another Markup Language 